\title{
Status Quo and Prospect of Colorectal Animal Models: Application of Transgenic Technology
}

\author{
Jiang Haili Liu Xuan Ji Qing Li Qi \\ Department of Medical Oncology, Shuguang Hospital, Shanghai University of Traditional \\ Chinese Medicine, Shanghai, China
}

Key Words

Transgenic technology $\cdot$ Animal models $\cdot$ Colorectal cancer

\section{Abstract}

Colorectal cancer (CRC) is one of the most common malignant diseases and brings about serious damage to the human body. Presently, the incidence of CRC ranks third among the malignant tumors, and its 5 -year survival rate is only about $50 \%$. In recent years, more and more studies have focused on the application of transgenic technology in CRC research in vivo, which involves a great number of genes significantly related to the occurrence and development of CRC. The core of transgenic technology refers to the host chromosome gene accepting exogenous genes, which integrate into the host chromosome expressing it stably, followed by transmission to the next generations. Here, we will present a brief review of the related technologies used in transgenic animal models and provide an overview of the application of transgenic animal models in CRC research.

(C) 2015 S. Karger AG, Basel

\section{Introduction}

In China, the incidence of colorectal cancer (CRC) is increasing year by year, with an increase by $104 \%$ in men and by $99 \%$ in women in the past 12 years in Shanghai [1]. The formation of CRC involves the activation of multiple proto-oncogenes and the inactivation of tumor suppressor genes, whose occurrence and development is a complex process 
Haili et al.: Status Quo and Prospect of Colorectal Animal Models: Application of Transgenic Technology

comprising multiple stages and multiple genes [2]. With the development of genetic engineering technology, CRC transgenic animal models play an important role in the study of CRC in vivo.

The common CRC animal models include spontaneous animal models, induced animal models and the homograft or xenograft tumor animal models. The spontaneous animal models are largely dependent on feed and environment, and the incubation periods of most of these tumors are so long that the established models cannot reflect the in-house characteristics of human tumors. Secondly, the induced animal models are built by way of inducing chemical compounds, which has been gradually abandoned because of the very low incidence of tumors. Thirdly, the homograft or xenograft tumor animal models refer to the transplantation of a portable tumor to the congener or heterogenic nude mice in vivo, including the following methods: cell cultivation method, organization cultivation method, and the renal subcapsular grafting method. Due to the congenital immunodeficiency of nude mice, experimental animal models have a high success rate, keeping the structure and function of original tumors. However, nude mice still have humoral immunity function, and, thus, they do not represent optimal experimental CRC animal models. At present, transgenic technology is invading many research fields, with broad application prospects in species transformation and animal trait changes. It is gratifying to note that transgenic technology is responsible for multiple breakthroughs in CRC research. The established CRC animal model using transgenic technology has advantages over breeding, and the etiology of this model is closer to the naturally occurring process of CRC. In general, transgenic technology can provide ideal animal models for CRC research on pathology, diagnosis and treatment [3]. Therefore, we next present a brief review of the related technologies used in transgenic animal models and provide an overview of the application of transgenic animal models in CRC research.

\section{Related Technologies of Transgenic Animal Models}

Currently, there are various types of transgenic technologies, including the microinjection method, sperm carrier method, somatic cell cloning method, retrovirus method, embryonic stem cell-mediated method and oocyte carrier method [4, 5]. The microinjection technology microinjects exogenous DNA into the former nucleus of a fertilized egg cell by a microscopic instrument [4]. The sperm carrier method incubates the exogenous gene with the live sperm, and then, the live sperm will take up the exogenous genes and be fertilized in vitro; finally, the transgenic mice model will be acquired $[6,7]$. The basic principle of the somatic cell cloning method is the same as that of the sperm carrier method. The retrovirus method is implemented through a variety of retrovirus vectors, which help genes transfer to germ cells [7]. The embryonic stem cell-mediated method is described in detail as follows: the exogenous gene is firstly imported into embryonic stem cells by transfection, then the screened-out embryonic stem cells which carry the exogenous genes stably are operated in series to obtain chimeric mice, and, finally, the gene knockout homozygous mice will be produced through mutual mating. With the oocyte carrier method, the exogenous genes are imported into former oocytes of the fertilized egg. After the exogenous genes have entered the oocytes, sperm chromatin gets into the egg cells. As long as the exogenous genes integrate, each cell will carry the exogenous genes from the division to the maturation process of the fertilized egg, which avoids generating the chimeric animals. In addition, the gene knockout mice model was established based on transgenic technology, whose core technology is to artificially delete tumor suppressor genes using the homologous recombination method and the Cre/loxP-induced conditional gene knockout method [8]. 
Haili et al.: Status Quo and Prospect of Colorectal Animal Models: Application of Transgenic Technology

\section{Application of the Transgenic Animal Model in CRC Research}

\section{$A P C^{\text {Min/+ }}$ Transgenic Animals}

The $A P C^{\text {Min/+ }}\left(C 57 B L / 6 J-A P C^{\text {Min/+ }}\right)$ mouse model is the classical animal model to study the development of CRC $[9,10]$. APC is a tumor suppressor gene, which is directly involved in the Wnt signaling pathways, controlling the conservative evolution mechanism of cells in the division process and organization of the mature embryo. Eighty-five percent of colon cancers have $A P C$ gene deletion or inactivation. $A P C$ mutation or deletion will lead to a $\beta$-catenin deposit in the nuclear, activated Wnt signaling pathways, stimulate downstream protooncogene cyclin D1 and, finally, cause cancer [11]. Li and colleagues [12] found that the adenomas of $\mathrm{APC}^{\mathrm{Min} /+}$ mice began to occur at 9 weeks in the intestinal tract, and, in the following 9-24 weeks, the glands continued to increase until the mice died. With the emergence of the $A P C^{M i n /+}$ mouse model, Hong et al. [13] prepared various kinds of animal models based on the mutation of the $A P C$ gene, including $A P C^{\Delta 716}, A P C^{\Delta 1309}, A P C^{1638 N}$ and others. The researchers also found that the locations of the intestinal tumors of the $A P C$ mice were different. The tumor of the $A P C^{M i n /+}$ mice can be located in the colon and small intestine. The tumor of $A P C^{\Delta 716}$ was mainly concentrated in the small intestine. The polyps of $A P C^{\Delta 716} C d x 2^{+/-}$ based on $A P C^{\Delta 716}$ were mainly located in the colon, but the reason remains unclear.

$A P C^{M i n /+}$ animal models have been widely used in intestinal cancer research; especially, their application for the study of drug effects and mechanisms has increased. With the development of various new research, Sohn et al. [14] have established the Min $\left(A P C^{+/-} \mathrm{Msh}^{-{ }^{-}-}\right)$ mouse model based on the $A P C^{M i n /+}$ mouse model and showed that Msh2 gene mutation or deletion can prompt mutation of the $A P C$ and the $p 53$ gene, which confirms that the Msh2 gene plays an important role in the development of intestinal cancer and provides the foundation for further research on the relationship between related genes and CRC. Similarly, Yamada et al. [15] established the $\operatorname{Min}\left(A P C^{M i n /+} M d r 1 a / b^{-/-}\right)$mouse model based on the $A P C^{M i n /+}$ mouse model and preliminarily elaborated the relationship between $M d r 1$ gene mutations and CRC. The mutation of the Mdr1 gene inhibited the occurrence of intestinal polyposis. The Mdr1 gene can make a $\beta$-catenin deposit in the nuclear, activated Wnt signaling pathways, leading to a tumor. Thus, $M d r 1$ gene deletion of the $\operatorname{Min}\left(A P C^{M i n /+} M d r 1 a / b^{-/-}\right)$mouse model can reduce the incidence of tumor in mice significantly. Niho et al. [16] have used the $A P C^{\Delta 1309}$ mouse model to confirm that $A P C$ gene mutations can inhibit the formation of small intestinal adenoid tumors when in a state of hyperlipidemia. Moreover, Akeus et al. [17] have successfully set up an $A P C^{\mathrm{Min} /+}$ knockout gene mouse model and identified the relationship between cancerassociated lymphocytes and the accumulation of gastrointestinal adenoma. They draw a preliminary conclusion that mutations and the deletion of $A P C^{M i n /+}$ in Wnt signaling pathways may affect the development of lymphatic system tumors, the number of $T$ cells and the accumulation of adenoma.

From the reviews mentioned above, we know that the $A P C^{\mathrm{Min} /+}$ mouse model has been widely used in intestinal cancer research. At the same time, some studies suggest that $A P C^{\text {Min/+ }}$ mouse models also include the obvious pathological changes in the digestive system, reproductive system, endocrine system, immune system and hematopoietic system. Therefore, the $A P C^{\text {Min/t+}}$ mouse models can be improved to adapt to each system disease research with all kinds of drug intervention or transgenic technology, and the $A P C^{\mathrm{Min} /+}$ mouse models can serve as a good model of 'integration' [18].

Tiam1 Transgenic Animals

The Tiam1 (T-cell lymphoma invasion and metastasis) gene is an induced factor in the invasion and metastasis of T-cell lymphoma [19]. Tiam1, with its high and positive expression in colon cancer cells, can act as a promoter in the invasion and metastasis of CRC. It was found 
Haili et al.: Status Quo and Prospect of Colorectal Animal Models: Application of Transgenic Technology

that the tumor cells expressing high Tiam1 have more phenotypes, a higher expression of $\beta$-catenin and vimentin, and a reduced $\mathrm{E}$-cadherin expression which can increase cell adhesion [20]. In order to explore the mechanisms of the Tiam1 gene leading to cancer, Yu et al. [20] have established a Tiam1/C1199-CopGFP transgenic mouse model using microinjection to research oncogenes and the mechanisms of invasion and metastasis of CRC. Compared to wild-type mice, the tumor volume of Tiam1 transgenic mice is bigger, and the invasion is stronger. Tiam1 likely accelerated the invasion and metastasis of CRC through the activation of the Wnt/ $\beta$-catenin signaling pathway. Tiam1 is associated with the differentiation and metastasis of CRC [21]. The mechanism of Tiam1 promoting CRC metastasis may also relate to epithelial mesenchymal transition. All the above results provide a reliable basis for further investigation into the mechanisms of Tiam1, which accelerated the invasion and metastasis of CRC.

\section{Cdc20 Transgenic Animals}

$C d c 20$, a cell cycle regulation factor, is indispensable to cell mitosis. Its main feature is to make the $A P C / C$ substrate degrade in an orderly manner during the process of mitosis to coordinate the process of mitosis. $A P C / C$, also known as cell cycle enzyme, is a kind of E3 ubiquitin-ligating enzyme and can make cell cycle proteins biodegradable [22]. Nilsson et al. [23] have produced $C d c 20^{\text {loxp/+ } A P C} C^{\mathrm{Min} /+}$ gene mutation mouse models. Firstly, they prepared $C d c 20^{\text {loxp } /+}$ gene mutant mice with the help of gene-targeting technology, then let the $C d c 20^{\text {loxp } /+}$ gene mutant mice and $A P C^{M i n /+}$ transgenic mice hybridize. The pathological type of intestinal tumor in this kind of mouse model is adenoma, whose malignant degree is higher than in the other models. A specific knockout homologous arm of the $C d c 20$ gene in the colon can accelerate the development of CRC. Pathological biopsy showed that the gland structure disorder has reached mucosal muscularis, with obvious cellular variation, nuclear atypia and hyperchromatism, and pathological fission visible in $C d c 20^{\text {loxp/+ }} A P C^{M i n /+}$ tumor tissue. $C d c 20$ transgenic animal models have laid a solid foundation for exploring the relationship between Cdc20 and the development of CRC and have well prepared for research on its molecular mechanism. Current research has shown that mutation of the $C d c 20$ gene can affect the development of CRC through the gene instability.

\section{MLH1 Transgenic Animals}

$M L H 1$, a mismatch repair gene [24], can ensure the accuracy of DNA replication. The carcinogenic effects of $M L H 1$ are mainly caused by the methylation of its promoter, followed by the inactivation of the DNA mismatch repair gene and the point mutation of DNA which cannot be repaired in time. This mechanism is thought to be an important pathogenesis of CRC [25]. Recent studies have shown that hereditary nonpolyposis CRC (HNPCC), an autosomal dominant genetic disease syndrome, can cause gene inactivation and lead to tumorigenesis mainly by making $C p G$ islands located in the $M L H 1$ gene promoter region methylate [26]. MLH1 knockout mice have been successfully applied in CRC research [27]. Zeng et al. [28] have successfully built an MLH1 knockout mouse model and found that the incidence of colon cancer in mice increased significantly, but the survival of mice was significantly reduced. They have also built a $M l h 1^{+/-}$heterozygous mouse model based on the MLH1 knockout mice model and further confirmed that the expression of decline or inactivation of the tumor suppressor gene DKK1 (an important molecule antagonist of the Wnt signaling pathway) is an important index of CRC.

\section{hMSH2 Transgenic Animals}

The basic function of $h M S H 2$, another mismatch repair gene, is to move insertion/deletion rings generated by the primary template in the case of repetitive DNA sequence slipping and 
Haili et al.: Status Quo and Prospect of Colorectal Animal Models: Application of Transgenic Technology

to check the single-base mismatch that escaped the correction of the read code in order to prevent the accumulation of spontaneous mutation and to guarantee the integrity and stability of the DNA replication [29]. The mechanism of $h M S H 2$-induced CRC is similar to that of MLH1. Methylation of the $h M S H 2$ gene promoter can cause the $M M R$ gene to express failure, which leads to CRC [30-32]. The $M M R$, an important repair system after replication, can maintain an accurate copy and control gene mutation [33]. In order to explore the effect of the hMSH2 gene in the development of CRC, Lowsky et al. [34] have successfully built a hMSH2 knockout mouse model. They found that the incidence of intestine adenoma in the $h M S H 2$ knockout mice was obviously higher than in normal mice. $h M S H 2$ genes may play a driving role in every link in the development of CRC.

About $90 \%$ of $H N P C C$ have an abnormal $h M S H 2$ and MLH1 gene expression [35]. DNA mismatch repair gene mutation or deletion played an important role in the pathogenesis of CRC. All the models presented above provided new insight for CRC research, guiding the clinical diagnosis and targeted therapy of CRC.

\section{Claudin-7 Transgenic Animals}

The Claudin-7 family protein is one of the main structural elements of intercellular tight junction proteins, whose expression has tissue specificity. Claudin-7 played an important role in maintaining intestinal epithelial cell polarity and in closely connecting the barrier function [36]. The mutation or abnormal expression of Claudin-7, as a potential tumor suppressor gene, is closely related to the occurrence of a variety of tumors [37]. In normal state, Claudin-7 is widely expressed in the colon and small intestine. In order to confirm the tumor suppressor function of this gene, Ding et al. [38] have established a Claudin-7 knockout mouse model by knockout Claudin-7 gene. They found mucosal epithelial cell shedding, vacuolation and inflammatory cell infiltration in the small intestines of knockout Claudin-7 gene mice. At the same time, $C$-fos, $C$-jun and $C O X-2$ showed significantly higher expression, and the expression of $N F-\kappa B, p 65, P-p 65$ and $P-I K B a t$ and the phosphorylation of histone $\mathrm{H} 3$ in the intestinal tract of the Claudin-7 knockout mice were also significantly increased [39]. Claudin-7 knockout mice stoped growing at day 4 and showed the obvious phenomenon of dehydration. Then they died at day 7; however, the controls were normal. After dissecting the intestines of these mice, inflammation and proliferation in the intestinal tract was obviously found [40]. Thus, we can draw the conclusion that Claudin-7 is a potential tumor suppressor gene in the development of CRC. It should be applied to further research inflammation in CRC and as a new target for CRC treatment.

\section{HLA-B27 Transgenic Animals}

$H L A-B 27$, one product of the human histocompatibility complex, is a specific diagnosing factor for ankylosing spondylitis. Recent research revealed that $H L A-B 27$ is closely related to CRC [40]. In 1995, Hammer et al. [41] have built an F344-B27 transgenic rat model which was an inbred line of the HLA-B27 transgenic rat. This transgenic rat model showed chronic inflammation in the intestinal tract. The inflammatory sites have the tendency of developing hyperplasia. The incidence of colorectal polyps is high, with the tendency of evolution from adenoma to carcinoma. The high expression of $H L A-B 27$ will increase the incidence of CRC, and early detection and elimination of HLA-B27 expression can reduce the risk of CRC.

\section{Microsatellite Instability Transgenic Animals}

Microsatellite instability (MSI) plays an important role in the development of CRC. It can be an important index to judge the prognosis of CRC [42]. A related study showed that the main tumorigenic mechanism is that MSI can cause microsatellite sequence mutations in the gene exon region and mutate the protein reading frame, producing abnormal peptide frag- 

Haili et al.: Status Quo and Prospect of Colorectal Animal Models: Application of
Transgenic Technology

ments and stimulating the body to produce an antitumor immune response [43]. The main pathological features of $M S I$ are as follows: the tumors are mainly located in the proximal colon, and mucous adenocarcinomas are often seen, with poor differentiation. However, currently, MSI animal models are still not established worldwide, which restricts research on its biological characteristics as well as on related treatments [44].

\section{Transgenic Animals}

$D C C$ is a suppressor gene of CRC, whose main functions are to increase the adhesion and contact ability among the cells [45]. The mutation or deletion of $D C C$ will lead to differentiation, metastasis and lymph node metastases of the cancer. $D C C$ can be an index for evaluating the prognosis of CRC. However, there is a lack of DCC transgenic mouse models [46]. In recent years, researchers have found that the cytoplasm of $D C C$ can be merged with the Sina protein of drosophila. Based on this, researchers have built a DCC/Sina transgenic drosophila and found that these two genes are expressed in drosophila eyes. The adjustment effect of the Sina protein to $D C C$ is achieved by the ubiquitin proteasome pathway $[47,48]$. The results of this former study inspired us to build $D C C$ transgenic mice models. The $D C C$ gene is expected to become important in the clinical diagnosis index of the invasion and metastasis of CRC and drive on drug research in CRC.

\section{Discussions}

The application of transgenic animal models in the research of CRC has made certain progress. $A P C^{\mathrm{Min} / \mathrm{+}}$ mice, a typical animal model, have been widely used in the research of CRC. The newly built models based on the $A P C^{\mathrm{Min} /+}$ mice model can also be applied to the research on other system cancers, so $A P C^{M i n /+}$ mice can be seen as the 'integration' model. A host of other transgenic animal models associated with CRC genes have been established and applied in CRC research.

Although the application of transgenic technology in CRC research has made some progress, the establishment of a transgenic animal model for specific signaling pathways is still lacking. Further research is needed to knock out a specific gene to directly induce CRC. Transgenic technology in the study of CRC has a lot of advantages; however, some reports showed that transgenic animal models have a high fatality rate, and it was often seen in transgenic animals that the targeted gene is not well expressed or eventually silenced [49]. We are looking forward to build better animal models in order to guide the research on the molecular mechanisms and biology of CRC.

\section{Acknowledgements}

This work was supported by the Experimental Animal Special Fund of Science and Technology Commission of Shanghai (No. 13140902500 and 14140901402); the National Natural Science Foundation of China (No. 81273958, 81473478, and 81303103); the Project of Shanghai Committee of Science and Technology (No. 13ZR1461000), and the 'Chen Guang' project supported by the Shanghai Municipal Education Commission and Shanghai Education Development Foundation (No. 13CG47). 
Haili et al.: Status Quo and Prospect of Colorectal Animal Models: Application of Transgenic Technology

\section{References}

1 Leung WK, Chen WQ, Gu L, Long D, Law WL: Distributions of colorectal cancer in two Chinese cities with contrasting colorectal cancer epidemiology. J Gastroenterol Hepatol 2015;11:628.

2 Jemal A, Bray F, Center MM, et al: Global Cancer statistic. CA Cancer J Clin 2011;61:69-90.

-3 Kather JN, Kroll J: Transgenic mouse models of corneal neovascularization: new perspectives for angiogenesis research. Invest Ophthalmol Vis Sci 2014;55:7637-7651.

4 Takahashi G, Gurumurthy CB, Wada K, Miura H, Sato M, Ohtsuka M: GONAD: Genome-editing via Oviductal Nucleic Acids Delivery system: a novel microinjection independent genome engineering method in mice. Sci Rep 2015;22:11406.

5 Boivin GP: Update on transgenic animal technology. Lab Anim (NY) 2015;44:209.

6 Yang YF, Ding YQ: The application and progress of transgenic technology in cancer research. Medical Rev 2004;10:1-3.

-7 Jo DH, Lee JH, Jun HJ, Kim J, Wen Q, Hoang MH, Yu YS, Kim JH, Lee SJ: Geneex pression profiles of primary retinal pigment epithelial cells from apolipoprotein E knockout and human apolipoprotein E2 transgenic mice. Genet Mol Res 2015;14:1855-1867.

-8 Liu V, Dietrich A, Kasparek MS, Benhaqi P, Schneider MR, Schemann M, Seeliger H, Kreis ME: Extrinsic intestinal denervation modulates tumor development in the small intestine of $\mathrm{APC}^{\mathrm{Min} /+}$ mice. See comment in PubMed Commons below. J Exp Clin Cancer Res 2015;34:39.

-9 Ignatenko NA, Gerner EW, Besselsen DG: Defining the role of polyamines in colon carcinogenesis using mouse models. J Carcinog 2011;16:10.

$\checkmark 10$ Kang JS, Kim TM, Shim TJ, et al: Modifying effect of diallyl sulfide on colon carcinogenesis in C57 BL/6J-APC ${ }^{\text {Min } /{ }^{+}}$ mice. Asian Pac J Cancer Prev 2012;13:1115-1118.

-11 Wang L, Zhang Q: Application of the APC ${ }^{\mathrm{Min} /+}$ mouse model for studying inflammation-associated intestinal tumor. Biomed Pharmacother 2015;71:216-221.

12 Ye ZJ, Zheng L, Zhang M, Tang FT, Wang JT, Geng JG, Jin Li: The biology characteristics of APC ${ }^{\mathrm{min} /+}$ colorectal precancerous mice. J Clin Exp Pathol 2011;27:393-395.

13 Hong SQ, Chen J, Lai MD: The application of Min gene mutant in the research of intestinal cancer. Genetic 2008; 30:277-282.

14 Sohn KJ, Choi M, Song J, et al: Msh2 deficiency enhances somatic Apcand p53 mutations in Apc ${ }^{-/-}$Msh2 ${ }^{-/-}$mice. Carcinogenesis 2003;24:217-224.

15 Yamada T, Mori Y, Hayashi R, et al: Suppression of intestinal polyposis in Mdr1-deficient APC ${ }^{\text {Min }}$ mice. Cancer Res 2003;63:895-901.

16 Niho N, Takahashi M, Kitamura T, Shoji Y, Itoh M, Noda T, Sugimura T, Wakabayashi K: Concomitant suppression of hyperlipidemia and intestinal polyp formation in Apc-deficient mice by peroxisome proliferator-activated receptor ligands. Cancer Res 2003;63:6090-6095.

17 Akeus P, Langenes V, von Mentzer A, et al: Altered chemokine production and accumulation of regulatory T cells in intestinal adenomas of $\mathrm{APC}^{\mathrm{Min} /+}$ mice. Cancer Immunol Immunother 2014;63:807-819.

18 Hu YT, Lu Y, Zhao HX, Hong SQ: Min disease 'integration' model. Anhui Med J 2011;32:1189-1192.

19 Wang YJ, Hu J, Zhao HY, Han HX: The relationship between Tiam1 and colorectal cancer cells EMT. Shijie Huaren Xiaohua Zazhi 2009;17:3337-3341.

20 Yu LN, Zhang QL, Li X, Hua X, et al: Tiam1 transgenic mice display increased tumor invasive and metastatic potential of colorectal cancer after 1,2-dimethylhydrazine treatment. PLoS One 2013;8:e73077.

21 Cottonham CL, Kaneko S, Xu L: MiR-21 and MiR-31 converge on TIAM1 to regulate migration and invasion of colon carcinoma cells. J Biol Chem 2010;285:35293-35302.

22 Shoji S, Muto Y, Ikeda M, et al: The zinc-binding region (ZBR) fragment of Emi2 can inhibit APC/C by targeting its association with the coactivator Cdc20 and UBE2C-mediated ubiquitylation. FEBS Open Bio 2014;4:689703.

23 Nilsson J, Yekezare M, Minshull J, et al: The APC/C maintains the spindle assembly check point by targeting Cdc20 for destruction. Nat Cell Biol 2008;10:1411-1420.

24 Auclair J, Vaissière T, Desseigne F, Lasset C, Bonadona V, Giraud S, Saurin JC, Joly MO, Leroux D, Faivre L, Audoynaud C, Montmain G, Ruano E, Herceg Z, Puisieux A, Wang Q: Intensity-dependent constitutional MLH1 promoter methylation leads to early onset of colorectal cancer by affecting both alleles. Genes Chromosomes Cancer 2011;50:178-185.

25 Miyakura Y, Tahara M, Lefor AT, et al: Haplotype defined by the MLH1-93G/A polymorphism is associated with MLH1 promoter hypermethylation in sporadic colorectal cancers. BMC Res Notes 2014;24:835.

26 Pussila M, Sarantaus L, Dermadi Bebek D, Valo S, Reyhani N, Ollila S, Paivarinta E, Peltomaki P, Mutanen M, Nystrmo M: Cancer-predicting gene expression changes in coloni mucosa of Western diet fed Mlh1 ${ }^{+/-}$Mice. PLoS One 2013;8:e76865.

27 Ji M, Sheng JQ, FuL, Mu H, Huang JS, Han M, Sun ZQ, Zhang H, Wu ZT, Zhang YH, Li SR: The research about hereditary nonpolyposis colorectal cancer mismatch repair gene MLH1 promoter methylation. J Gastroenterol Hepatol 2008;17:291-293.

28 Zeng JR, Ruan ZM, Mo LF, Chen H: Meta-analysis the relationship between MLH1 gene promoter methylation and colon cancer. Shijie Huaren Xiaohua Zazhi 2013;21:4204-4209. 
29 Wang CF, Zhou XY, Zhang TM, Sun MH, Xu Y, Shi DR: The mRNA mutation analysis of MLH1 and MSH2 and the diagnosis of HNPCC of colorectal cancer. J Chin Med Genet 2006;23:32-36.

-30 Yan ZH, Cui LH, Wang XH, et al: Comparative study of mutations in SNP loci of K-RAS, hMLH1 and hMSH2 genes in neoplastic intestinal polyps and colorectal cancer. World J Gastroenterol 2014;20:18338-18345.

-31 Li G, Hu F, Yuan F, Fan J, et al: Intronic and promoter polymorphisms of hMLH1/hMSH2 and colorectal cancer risk in Heilongjiang Province of China. J Cancer Res Clin Oncol 2015;6141-6143.

32 Wang Y, Li D, Li X, et al: Prognostic significance of hMLH1/hMSH2 gene mutations and hMLH1 promoter methylation in sporadic colorectal cancer. Med Oncol 2014;31:39.

33 Jin HY: The progress research of hMSH2 polymorphism and the susceptibility of tumor. J Gastrointest Surg 2003;6:243-246.

-34 Lowsky R, DeCoteau JVF, Reitmair AH, Ichinohasama R, Dong WF, Xu Y, Mak TW, Kadin ME, Minden MD: Defects of the mismatch repair gene MSH2 are implicated in the development of murine and human lymphoblastic lymphomas and are associated with the aberrant expression of rhombotin-2 (Lmo-2) and Tal-1 (SCL). Blood 1997;89:2276-2282.

35 Cai Q, Sun MH, Fu G, et al: Mutation investigation of hMLH1 and hMLH2 genes in hereditary nonpolyposis colon cancer families. J Chin Pathol 2003;323-328.

-36 Li X, Li Y, Qiu H, Wang Y: Downregulation of claudin-7 potentiates cellular proliferation and invasion in endometrial cancer. Oncol Lett 2013;6:101-105.

37 Bhat AA, Pope JL, Smith JJ, Ahmad R, Chen X, Washington MK, Beauchamp RD, Singh AB, Dhawan P: Claudin-7 expression induces mesenchymal to epithelial transformation (MET) to inhibit colon tumorigenesis. Oncogene 2014;11:465-578.

38 Ding L, Lu Z, Foreman O, Tatum R, Lu Q, Renegar R, Cao J, Chen YH: Inflammation and disruption of the mucosal architecture in claudin-7-deficient mice. Gastroenterology 2012;142:305-315.

39 Ding L, Gao H, Chen Y: The mechanism research of claudin-7 knockout mice induced intestinal inflammation and carcinogenic. J Surg 2013;28:120-124.

40 Alcalay M, Bontoux D, Maire P, Matuchansky C, Alcalay D, Tanzer J: HLA-B27 and colorectal cancer. N Engl J Med 1982;307:443-444.

41 Hammer RE, Richardson JA, Simmons WA, White AL, Breban L, Taurof JD: High prevalence of colorectal cancer in HLA-B27 transgenic F344 rats with chronic inflammatory bowel disease. J Investig Med 1995;43:262-268.

42 Je EM, Yoo NJ, Lee SH: Mutational and expressional analysis of MLL genes in gastric and colorectal cancers with microsatellite instability. Neoplasma 2013;60:188-195.

-43 Karahan B, Argon A, Yidirim M, Vardar E: Relationship between MLH-1, MSH-2, PMS-2, MSH-6 expression and clinicopathological features in colorectal cancer. Int J Clin Exp Pathol 2015;8:4044-4053.

44 Kohonen-Corish MR, Daniel JJ, te Riele H, Buffinton GD, Dahlstrom JE: Susceptibility of Msh2-deficient mice to inflammation-associated colorectal tumors. Cancer Res 2002;62:2092-2097.

-45 Djansugurova L, Zhunussova G, Khussainova E, Iksan O, Afonin G, Kaidarova D, Parker MI: Association of DCC MLH1, GSTT1, GSTM1, and TP53 gene polymorphisms with colorectal cancer in Kazakhstan. Tumour Biol 2015;36:279-289.

46 Broutier L, Castets M: DCC, come back of a suppressor gene in colorectal cancer. Med Sci (Paris) 2012;28: 465-468.

47 Yang HJ, Ding YQ: The application of transgenic animals in colorectal cancer research. Med Rev 2001;12:736737.

48 Hu G, Zhang S, Vidal M, Bael JL, Xu T, Fearon ER: Mammalian homologs of seven in absentia regulate DCC via the ubiquitin proteasome pathway. Gene Dev 1977;11:2701-2714.

49 Lin HY, Ying RT, Xi T, Xu HM: The research progress of transgenic mouse models used in cancer research. Chin J Pharmacol 2007;23:4-8. 